\title{
Total Saikosaponins of Bupleurum yinchowense reduces depressive, anxiety-like behavior and increases synaptic proteins expression in chronic corticosterine-treated mice
}

\author{
Xiuping Sun ${ }^{1}$, Xianglei Li', Ruile Pan², Yanfeng Xu', Qiong Wang ${ }^{3}$ and Mingjing Song ${ }^{1 *}$
}

\begin{abstract}
Background: Bupleurum yinchowense Shan et $Y . L i$ is widely used to treat depressive and anxiety disorders for hundreds of years in China. Total saikosaponins (TSS) is the major ingredient of Bupleurum yinchowense. A-amino-3hydroxy-5-methyl-4-isoxazolepropionic acid (AMPA) receptor and subsequent mammalian target of rapamycin (mTOR) signaling is responsible for synaptic maturation and may contribution to the synaptic alteration underlying depression. The aim of the study was to investigate the antidepressant-like and anxiolytic effect of TSS in chronic corticosterone-treated mice. The effect of TSS on synaptic proteins expression and AMPA receptor-mTOR signaling pathway alteration was also evaluated.

Methods: Dose-response effect of TSS $(12.5,25,50 \mathrm{mg} / \mathrm{kg})$ was investigated in forced swim test (FST) in ICR male mice. In the chronic corticosterine-treated model, TSS was given intragastrically once a day for 2 weeks and continued through the behavior testing period. Behavior tests and AMPA receptor related signaling pathway were investigated.

Results: TSS ( 25 and $50 \mathrm{mg} / \mathrm{kg}$ ) decreased the immobility time in the FST when compared with the control group. TSS $(25 \mathrm{mg} / \mathrm{kg}$ ) showed antidepressant-like and anxiolytic effects in the chronic corticosterone treatment model in mice. TSS increased hippocampal synaptic proteins (synapsin-1 and postsynaptic density protein 95) expression. Immunohistochemistry analysis showed that TSS significantly increased the synapsin-1 expression in CA3 of hippocampus. TSS also increased hippocampal phosphorylation expression of GluR1 Ser 845 (AMPA receptor subunit) and its downstream regulators extracellular signaling-regulated kinase (ERK), protein kinase B (Akt) and mTOR.

Conclusion: TSS produces antidepressant-like and anxiolytic effects and increases synaptic proteins expression which may be mediated by induction of AMPA receptor and subsequent mTOR signaling pathway.
\end{abstract}

Keywords: Depression, Anxiety, AMPA receptor, Corticosterone, Bupleurum yinchowense, Synaptic protein

\footnotetext{
*Correspondence: smjf2017@126.com

${ }^{1}$ Comparative Medical Center, Beijing Key Laboratory for Animal Models of

Emerging and Reemerging Infectious Diseases, Peking Union Medical

College (PUMC) \& Institute of Laboratory Animal Science, Chinese Academy

of Medical Science(CAMS), PanjiayuanNanli No. 5, Beijing 100021, China

Full list of author information is available at the end of the article
} 


\section{Background}

Bupleurum yinchowense Shan et $Y$. Li, a widely used Chinese medicine, was first documented in the "Shennong's Herbal", which is the oldest Chinese materia medica monographs. It was recorded that Bupleurum yinchowense has action of soothing liver and was used to treat depressive and mood related disorders [1]. Our preliminary experiments indicated that total saikosaponins (TSS) from Bupleurum yinchowense produced antidepressant-like effect in the forced swim test (FST), tail suspension test in normal mice. Meanwhile, TSS exhibited antidepressant-like effect in chronic mild stress model in mice [2]. However, the underlying mechanisms of these effects are not yet clear.

Recently, clinical and basic studies demonstrate that depression is associated with synaptic dysfunction in important regions such as prefrontal cortex and hippocampus [3]. Simultaneously, accumulating evidence has shown that A-amino-3-hydroxy-5-methyl-4-isoxazolepropionic acid (AMPA) receptor and subsequent mammalian target of rapamycin (mTOR) signaling way is responsible for the induction and maintenance of synaptic plasticity and may contribution to the synaptic alteration underlying depression $[4,5]$. Chronic AMPA potentiators and AMPA treatment produce antidepressant-like effect and stimulate mTOR protein transduction signaling way [6]. The mTOR singaling is a downstream intracellular signal pathway which controls the synthesis of synaptic proteins and contributes to synaptogenesis [7]. Ketamine fast induction of mTOR singaling and synaptogenesis may be dependent on AMPAR activation [8]. Sarcosine, an endogenous amino acid, exhibits antidepressant-like effects by activating AMPAR-mTOR signaling pathway [9]. These data suggest that AMPA receptors and subsequent mTOR signaling may be a potential therapeutic target for emerging antidepressant-like agent actions.

Chronic stress-mediated elevated levels of circulating glucocorticoids (corticosterone, CORT, in rodents) are widely believed to be involved in the pathophysiology of depression [10]. Chronic and direct administration of corticosterone to male rodents has been used as a model to induce anxiety/depression-like behavior [11, 12]. In such models, hippocampal dendritic spines and spine synapses were decreased which is accompanied by a loss of key synaptic proteins such as postsynaptic density protein 95 (PSD-95), and the presynaptic protein synapsin-1 [12]. Interestingly, some findings indicated CORT exerted its impact on synaptic plasticity by modulating AMPA receptor [13].

Our recent research showed that TSS induced protective effect in corticosterone-induced damage in PC12 cells [14]. On the basis of above findings, we hypothesize that chronic CORT treatment-induced depressive and anxiety like behaviour of mice is caused by dysregulation of AMPA receptor and subsequent mTOR signaling pathway and treatment with TSS might ameliorate these behavioural and molecular changes.

\section{Methods \\ Preparation of Total Saikosaponins (TSS)}

TSS was extracted as previous prescribed [14]. Five main monomeric compounds in TSS were determined using HPLC and the content of Saikosaponins a, c, d, e and $\mathrm{f}$ was $10.12 \%, 2.84 \%, 14.13 \%, 1.52 \%$ and $2.14 \%$, respectively.

\section{Animals}

ICR and C57BL/6J male mice (23-25 g) were obtained from Beijing HFK Bioscience CO., LTD. The animals were group-housed (5 per cage) under the standard conditions $\left(25{ }^{\circ} \mathrm{C}\right.$ with a $12 \mathrm{~h} / 12 \mathrm{~h}$ light/dark cycle) and received standard food and tap water ad libitum. The experiments were approved by the ethical committee for the use of experimental animals of the Institute of Laboratory Animal Sciences (No: ILAS-PG-2015-011).

\section{Drugs}

Citalopram was purchased from the National Institutes for Food and Drug Control (Beijing, China). Citalopram were dissolved in distilled water. Corticosterone (CORT, from Sigma, 27,840) was dissolved in vehicle $(0.45 \%$ hydroxypropyl- $\beta$-cyclodextrin, $\beta-C D$ from Binzhou Zhiyuan Bio-Technology, China).

\section{Experiment 1 dose-response effect of TSS in the FST}

Forty-one ICR male mice were divided randomly into control (distilled water, $n=10)$, TSS $(12.5 \mathrm{mg} / \mathrm{kg}, n=10)$, TSS (25 mg/kg, $n=10)$ and TSS (50 mg/kg, $n=11)$ groups. TSS or distilled water was given orally $1 \mathrm{~h}$ before testing. FST is the most popular behavioral test to evaluate depression-like behavior in rats and mice, with a high predicative validity for screening antidepressant drugs [15]. According to the method described by Porsolt, the apparatus consisted of a plastic cylinder (height $20 \mathrm{~cm}$, diameter $12 \mathrm{~cm}$ ) filled with water $\left(25^{\circ} \mathrm{C}\right)$ to a depth of $15 \mathrm{~cm}$. Mice were individually placed into the cylinder for $6 \mathrm{~min}$. The immobility time was recorded during the last 4-min interval of the test by trained observers who were blind to the treatment of mice. Each mouse was judged to be immobile when it ceased struggling and made only small movements to keep its head above the water [15].

\section{Experiment 2 anxiolytic, antidepressant-like effects and biochemical alteration of TSS in chronic corticosterone- treated mice}

To assess the depressive, anxiety behavior and molecular alteration of TSS administration in chronic corticosteronetreated mice, male C57BL/6 J mice were randomly divided 
into 4 groups ( $n=8-9$ per group): Control $(n=9)$, CORT $(n=8)$, citalopram $(10 \mathrm{mg} / \mathrm{kg}, n=8))$ and TSS $(25 \mathrm{mg} / \mathrm{kg}$, $n=9)$. Corticosterone $(35 \mu \mathrm{g} / \mathrm{ml}$ equivalent to $5 \mathrm{mg} / \mathrm{kg} /$ day) was delivered for 35 days in drinking water in opaque bottles and continued through the behavior testing period. Control animals received vehicle $(\beta-C D)$ in drinking water during the entire experiment. After 3 weeks of drug-free exposure to CORT administration, TSS was given intragastrically once a day for 14 days and continued through the behavior testing period. Citalopram was used as positive control. Open field test (OFT), elevated plus maze (EPM) test, novelty suppressed feeding test (NSFT) and forced swim test (FST) were performed to assess anxiousdepressive like behavior (Fig. 1).

\section{Behavior tests}

\section{Open field test (OFT)}

Anxiety behavior and motor activity was quantified in an open field paradigm $(50 \times 50 \times 50 \mathrm{~cm})$ using a computerized video-tracking system (EthoVision XT,Noldus). Animals naturally tend to avoid the central parts of open field. Anxiety state was assessed by the time spent in the center area of open field. In addition, OFT is routinely used to assess spontaneous locomotor activity in rodents [16].The open field was divided into center, transition and border arena. The behavior experimenters were blind to the treatment of mice. Each mouse was gently placed in the center of the box. The total distance moved, time in the center area and velocity were recorded for $5 \mathrm{~min}$ and analyzed by automated system software to evaluate the locomotor activity and anxietyrelated behavior.

\section{Elevated plus maze (EPM) test}

EPM test has widely been used for screening anxiolytic drugs. The animals spontaneously prefer the closed arms to open ones. Anxiety state was assessed by the percentage of open arm entries and percentage of time spent on the open arms [17].The maze was made of two open

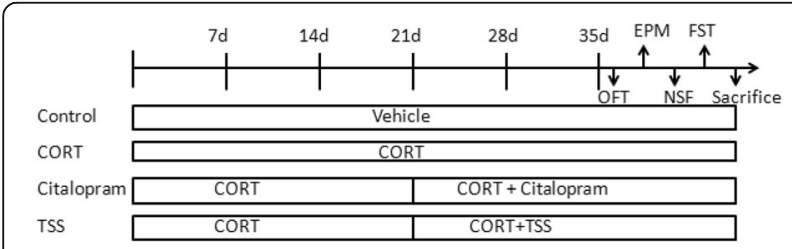

Fig. 1 The schematic representation of experiment 2 design. Male C57BL/6 J mice were divided 4 groups: Control, CORT, Citalopram $(10 \mathrm{mg} / \mathrm{kg})$ and TSS $(25 \mathrm{mg} / \mathrm{kg})$. Vehicle and corticosterone were delivered for 5 weeks and continued through the behavior testing period. After 3 weeks of drug-free exposure to CORT administration, TSS and citalopram were given intragastrically once a day for 2 weeks and continued through the behavior testing period. OFT, EPM, NSFT and FST were performed. After behavior tests, the mice were sacrificed for western blot essay or immunohistochemistry analysis arms $(30 \mathrm{~cm} \times 5 \mathrm{~cm})$ and two closed arms $(30 \mathrm{~cm} \times$ $5 \mathrm{~cm} \times 15 \mathrm{~cm}$ ) and it was elevated $50 \mathrm{~cm}$ above the floor. The intersection of the arms was a central platform $(5 \mathrm{~cm} \times 5 \mathrm{~cm})$. The behavior experimenters were blind to the treatment of mice. The mouse was placed in the central platform with its head facing an open arm. After 5 min of free exploration, the mouse may be moved out of the maze and back into its home cage. The number of closed arm entries, number of open arm entries, and time spent on the open arms were recorded and analyzed by automated computer software (EthoVision $\mathrm{XT}$,Noldus). The percentage of open arm entries (\%OE $=100 \times$ open arm entries / total entries) and percentage of time spent on the open arms $(\% \mathrm{OT}=100 \times$ time spent on open arms / (time spent on open arms + time spent on closed arms) were calculated.

\section{Novelty suppressed feeding test (NSFT)}

NSFT has been used extensively to study anxiety and depression related behaviors since it is sensitive to anxiolytic and chronic antidepressant treatments [18].The apparatus consisted of a plastic box $(50 \mathrm{~cm} \times 50 \mathrm{~cm})$. After $24 \mathrm{~h}$ of food deprivation, a single pellet of food was placed in the center of the box. The animal was placed in a corner of the box and the latency to bite was recorded for $5 \mathrm{~min}$ by trained observers who were blind to the treatment of mice. The home-cage food consumption during the subsequent 5 min was measured.

\section{Western blotting analysis}

At $24 \mathrm{~h}$ following the behavior testing, animals were killed by decapitation, brains were removed and hippocampus was dissected out for western blot experiments. The dissected tissue was made in the RIPA lysis buffer with protease and phosphatase inhibitor cocktail (Cell Signaling Technology). The protein concentration in each sample was determined using a Pierce BCA protein assay kit. The proteins were separated on SDS-PAGE gel and transferred onto a nitrocellulose membrane. The membranes were blocked with the blocking reagent for $2 \mathrm{~h}$ and incubated at $4{ }^{\circ} \mathrm{C}$ overnight with the primary rabbit antibodies against phospho-mTOR (Ser-2448), phosohpo-ERK1/2 (Thr202/Tyr204), phospho-Akt (Ser-473), synapsin-1, PSD-95 (Cell Signaling Technology), phospho-GluR1 (Ser-845) (Abcam). Membranes were subsequent incubated for $1 \mathrm{~h}$ at room temperature in goat anti-rabbit HRP-conjugated secondary antibody (1:5000 in TBS, ZSGB-BIO, China). Immunoreactive bands were visualized using enhanced chemiluminescent kits (Santa Cruz).

\section{Immunohistochemistry analysis}

For immunohistochemistry, mice were anesthetized with sodium pentobarbital $(50 \mathrm{mg} / \mathrm{kg}$ body weight, i.p.) and perfused with a $4 \%$ paraformaldehyde solution. Tissues were 
routinely processed in paraffin blocks. Immunohistochemical staining was performed according to Polink-2 plus System kit (ZSGB-BIO, China). Sections were blocked in $10 \%$ normal goat serum and were subsequently incubated in synapsin-1primary antibody solution $(1: 1000)$ at $4{ }^{\circ} \mathrm{C}$ overnight. Sections were washed in PBS with $0.3 \%$ Triton $\mathrm{X}$ 100 and incubated in goat anti-rabbit secondary antibody (1:1000) for $1 \mathrm{~h}$ at room temperature. Images were taken with a BX40 microscope with an attached CCD camera system (Olympus, Tokyo, Japan). Image quantification was analyzed using Image-pro Plus software.

\section{Statistical analysis}

SPSS 20 was used for statistical analysis. Differences among means were analyzed using one-way analysis of variance (ANOVA) followed by the Student-NewmanKeuls test. All results are presented as mean \pm standard error of the mean (SEM). $P<0.05$ was considered statistically significant for all data.

\section{Results}

TSS produced antidepressant-like effect in the FST in mice As shown in Fig. 2, TSS, at dose of 25 and $50 \mathrm{mg} / \mathrm{kg}$, decreased the immobility time in the FST when compared with the control group $(\mathrm{F}(3,37)=7.512, P<0.01 ; P<0.05)$. Dose of $12.5 \mathrm{mg} / \mathrm{kg}$ did not produce significant effect.

TSS produced antidepressant and anxiolytic effects in the chronic cortiocosterone-treated mice Open field test

As shown in Fig. 3a, b, c, no significant difference in the distance moved and velocity was found between CORT

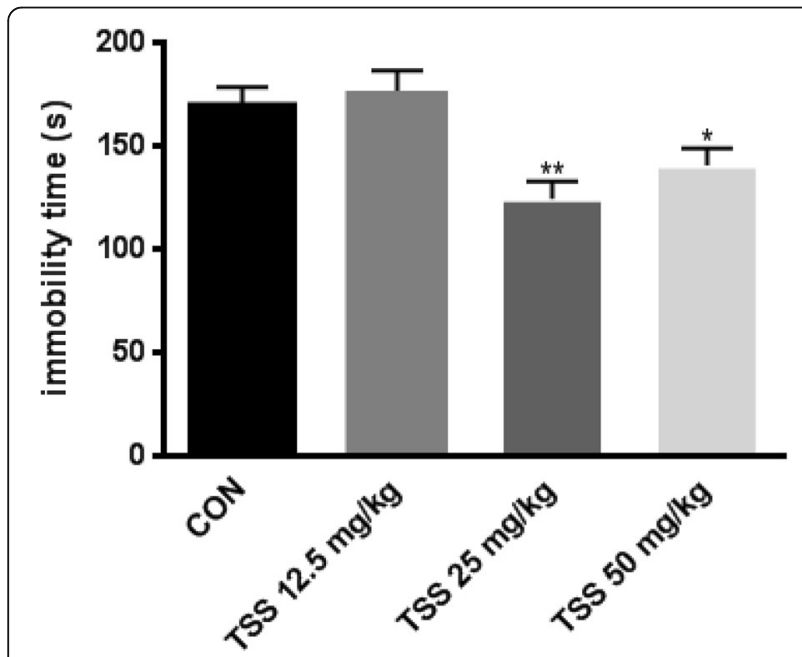

Fig. 2 Effects of TSS (12.5、25、50 mg/kg) on the forced swim test. Data were analyzed by one-way ANOVA followed by StudentNewman-Keuls test. Each bar represents the mean \pm SEM $(n=10-11)$. ${ }^{*} P<0.01,{ }^{*} P<0.05$, as compared with the control group group and control group $(\mathrm{F}(3,30)=4.314$, both $P>0.05)$. Citalopram significantly increased the velocity and distance moved compared with CORT group (both $P<0.01$ ). TSS treatment did not produce any change in the distance moved and velocity (both $P>0.05$ ). Both citalopram and TSS significantly increased the center time in the OFT which is decreased by CORT treatment $(F(3,30)=3$. 534,both $P<0.01$ ).

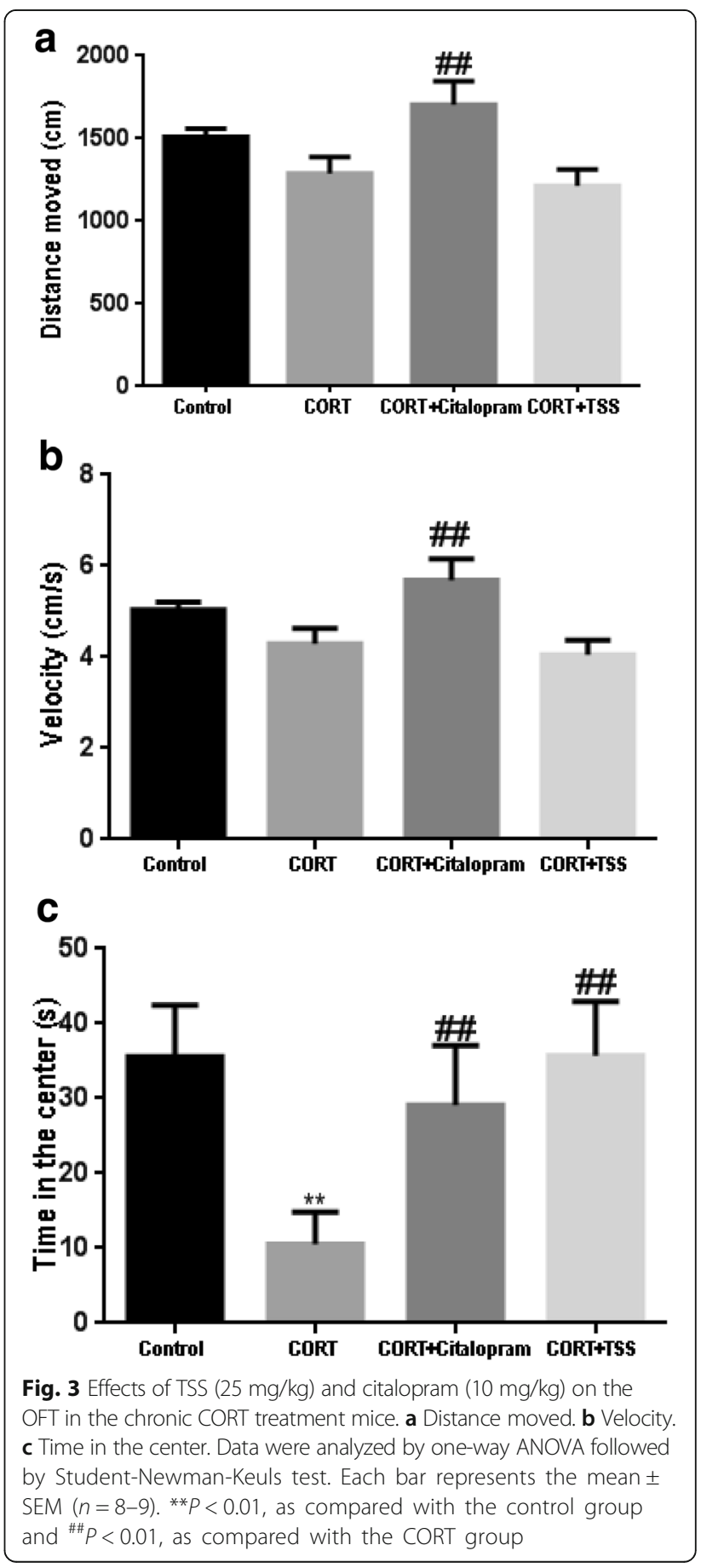




\section{Elevated plus maze test}

As shown in Fig. $4 \mathrm{a}$ and b, chronic corticosterone treatment induced significant reduction in the percentage of open arm entries and percentage of time on the open arms $(\mathrm{F}(3,30)=3.527, \quad P<0.01 ; \mathrm{F}(3,30)=2.913, P<0.05)$ compared with control group. Both citalopram and TSS treatment reversed chronic CORT treatment induced changes in the EPM test (both $P<0.05$ ).

\section{Novelty suppressed feeding test}

As shown in Fig. 5a and b, chronic corticosterone treatment significantly increased the latency time to feed in the NSF test with respect to the control group $(F(3,30)$ $=6.927, P<0.01)$. Both citalopram and TSS significantly reduced the latency time (both $P<0.01$ ). No differences in home-cage feed consumption were detected (both $P>0.05)$.

\section{Forced swim test}

As shown in Fig. 6, chronic corticosterone treatment significantly decreased the immobility time in the FST

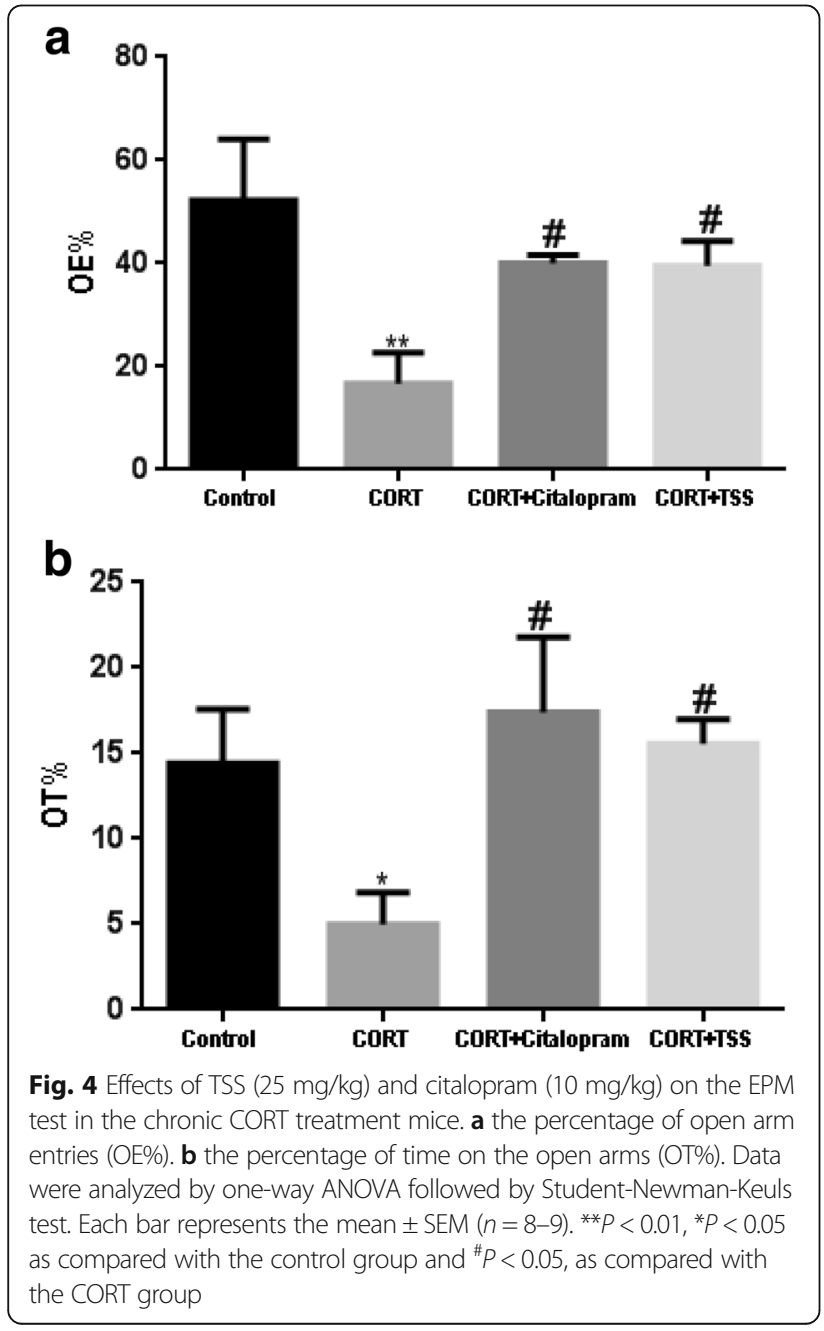

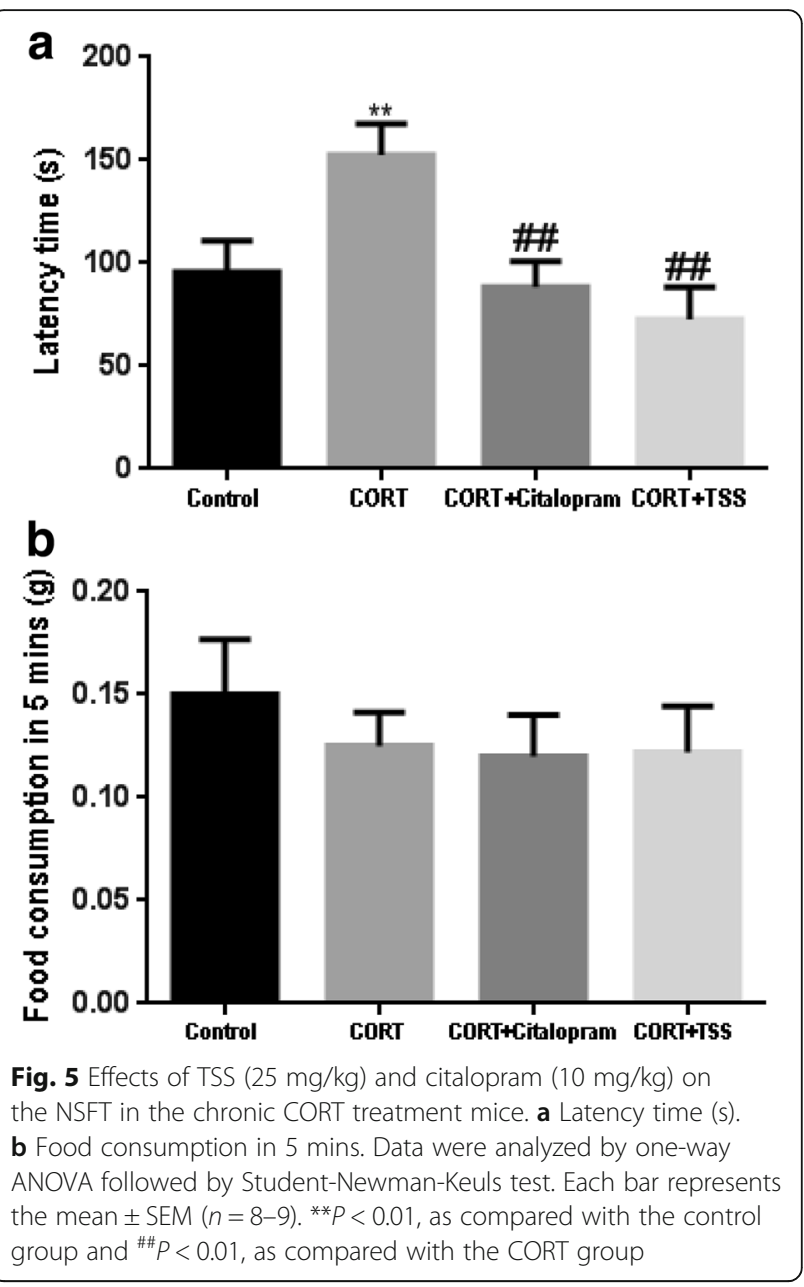

compared with control group $(F(3,30)=6.547, P<0.01)$. Both citalopram and TSS significantly increased the immobility time (both $P<0.01$ ).

TSS improved AMPA receptor-mTOR signaling pathway and synaptic proteins expression in the hippocampus of chronic corticosterone-treated mice TSS improved AMPA receptor-mTOR signaling pathway As shown in Fig. 7a, chronic corticosterone treatment significantly decreased the phosphorylation of hippocampal GluR1 Ser845 in the hippocampus in chronic corticosterone-treated mice $(\mathrm{F}(3,8)=48.83, \quad P<0.01)$, which was reversed by the citalopram and TSS treatment (both $P<0.01$ ). Total GluR1 level remained unchanged. Similarly, the results in Fig. 7b, c, d showed chronic corticosterone treatment significantly decreased the phosphorylation of mTOR, ERK and Akt in the hippocampus of chronic corticosterone-treated mice $(\mathrm{F}(3,8)=46.636 ; \mathrm{F}(3,8)=22.880 ; \mathrm{F}(3,8)=29.458$, both $P$ $<0.01)$. Citalopram and TSS treatment significantly increased the p-Akt (both $P<0.01)$, p-ERK $(P<0.05, P<0$. $01)$ and $\mathrm{p}-\mathrm{mTOR}$ level $(P<0.01, P<0.05)$. 


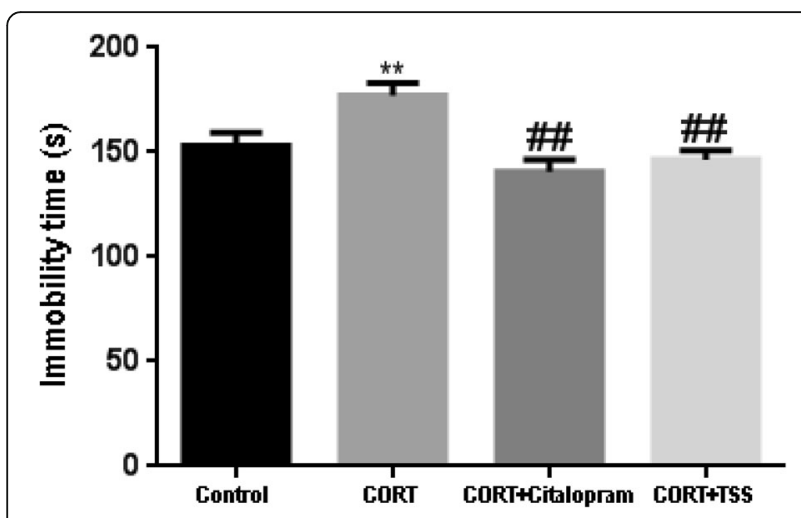

Fig. 6 Effects of TSS $(25 \mathrm{mg} / \mathrm{kg})$ and citalopram $(10 \mathrm{mg} / \mathrm{kg})$ in the FST in the chronic CORT treatment mice. Data were analyzed by one-way ANOVA followed by Student-Newman-Keuls test. Each bar represents the mean $\pm \operatorname{SEM}(n=8-9)$. ${ }^{* *} P<0.01$, as compared with the control group and ${ }^{\# \# P}<0.01$, as compared with the CORT group

\section{TSS improved synaptic proteins expression}

As shown in Fig. 7e, f, chronic corticosterone treatment significantly decreased synapsin-1 and PSD-95 level in the hippocampus in chronic corticosterone-treated mice $(\mathrm{F}(3,8)=37.245 ; \quad \mathrm{F}(3,8)=30.224 ;$ both $P<0.01)$. Citalopram and TSS treatment significantly increased synapsin1 and PSD-95 level (both $P<0.01$ ).

\section{Immunohistochemical analysis of synapsin-1 in the} hippocampus of chronic corticosterone-treated mice To further assess the effect of TSS on the expression of synapsin-1 in the hippocampus of chronic corticosteronetreated mice, immunohistochemical analysis was performed. Consistent with western blotting analysis results, as shown in Fig. 8, in the Cornu Amonis (CA1) (a) and CA3 (b), chronic CORT treatment mice displayed significantly less levels of synapsin-1, compared with control group $(\mathrm{F}(3,8)=16.269 ; \mathrm{F}(3,8)=22.305$; both $P<0.01)$. In the dentate gyrus (DG) region $(\mathbf{c})$, chronic CORT treatment did not cause a significant decrease in synapsin-1 expression $(\mathrm{F}(3,8)=4.563, P>0.05)$. TSS significantly increased the synapsin-1 expression in the CA3 region $(P<$ 0.01 ), but not in the CA1 and DG of hippocampus (both $P>0.05)$. Citalopram significantly increased the synapsin1 expression in the CA1 and CA3 region $(P<0.01, P<0$. $05)$. No significant change was observed after citalopram treatment in the DG region $(P>0.05)$.

\section{Discussion}

The present study indicates TSS exerts antidepressantlike and anxiolytic effects that are common to citalopram in chronic corticosterone-treated mice. Moreover, TSS increases synaptic proteins (synapsin-1 and PSD-95) which may be mediated by activation of AMPA receptor and mTOR signaling pathway. Dose-response assessment shows TSS produces antidepressant-like effect in the FST both in 25 and $50 \mathrm{mg} / \mathrm{kg}$ CORT-treated mice. Notably, the dose of $25 \mathrm{mg} / \mathrm{kg}$ presents maximum antiimmobility effect in the FST. Our previous work has shown that TSS at dose of $25 \mathrm{mg} / \mathrm{kg}$ was more efficacious than that of $50 \mathrm{mg} / \mathrm{kg}$ at different time point post the last TSS administration [19]. Previous study also indicated saikosaponin at dose of $25 \mathrm{mg} / \mathrm{kg}$ produced antidepressant-like effects in the chronic mild stress model [20]. Herein, the dose of $25 \mathrm{mg} / \mathrm{kg}$ was selected to further research.

To evaluate behavioral changes, in the present study, OFT, EPM, NSFT and FST were used to assess anxiousdepressive like behavior of the chronic corticosterone treatment model in mice. Our study showed that 5 week administration of CORT at dose of $35 \mu \mathrm{g} / \mathrm{ml}$ per day induced depressive like behavior as reflected by the increase in the immobility time in the FST. This model also exerted anxiety behavior as assessed by the decrease in the center area in the OFT and by the decreased percentage of open arm entries and percentage of time spent on the open arms in EPM test, as well as by the increased latency to feed in the NSFT. Thus, in this study, an anxiety-depressive model was successfully modeled by chronic treatment of CORT. These behavior results obtained from our study are consistent with previous findings $[11,21,22]$.

Depressive and anxiety disorder commonly occur together in clinic [23]. Clinic research has shown that more than $70 \%$ patients with depressive disorder present symptoms of anxiety disorder [24]. Co-morbid depression and anxiety increase suicide risk and contribute to treatment resistance [25]. The selective serotonin reuptake inhibitors (SSRIs) are usually used to treat comorbid depression with anxiety, although there are drawbacks including sexual and weight gain. In the present study, it is the first time to detect the anxiolytic and antidepressant-like effect of TSS in chronic CORT treatment model by behavior tests such as OFT, EPM, NSFT and FST. Citalopram, a classic prototypical selective serotonin reuptake inhibitor was the positive control. Our results showed that both TSS and citalopram exerted anxiolytic effect, as measured by a significant increase of time spent in the center of the open field and the increased percentage of open arm entries and percentage of time spent on the open arms in the EPM test, as well as by the decreased latency to feed in the NSFT. Furthermore, TSS was effective in reducing the immobility time in the FST, suggesting an antidepressant-like effect. Since locomotor activity may lead to false positive or negative effects in the FST, OFT was used to assess the locomotor activity of animals. Our research has shown that TSS did not produce significant changes in locomotion activity in the OFT. Therefore, the effect of TSS in the FST was not based on the unspecific action 


\section{a}
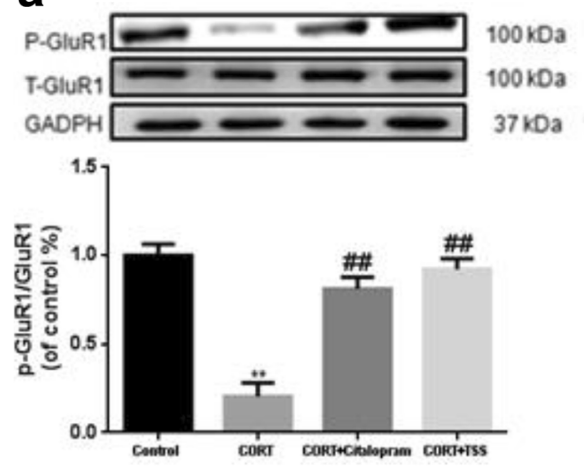

C
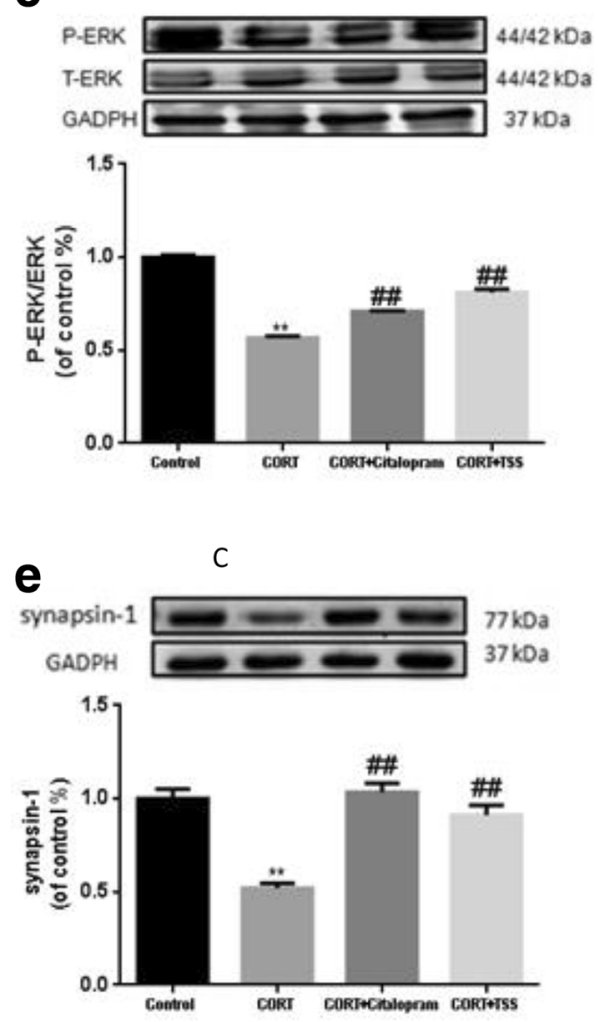

E
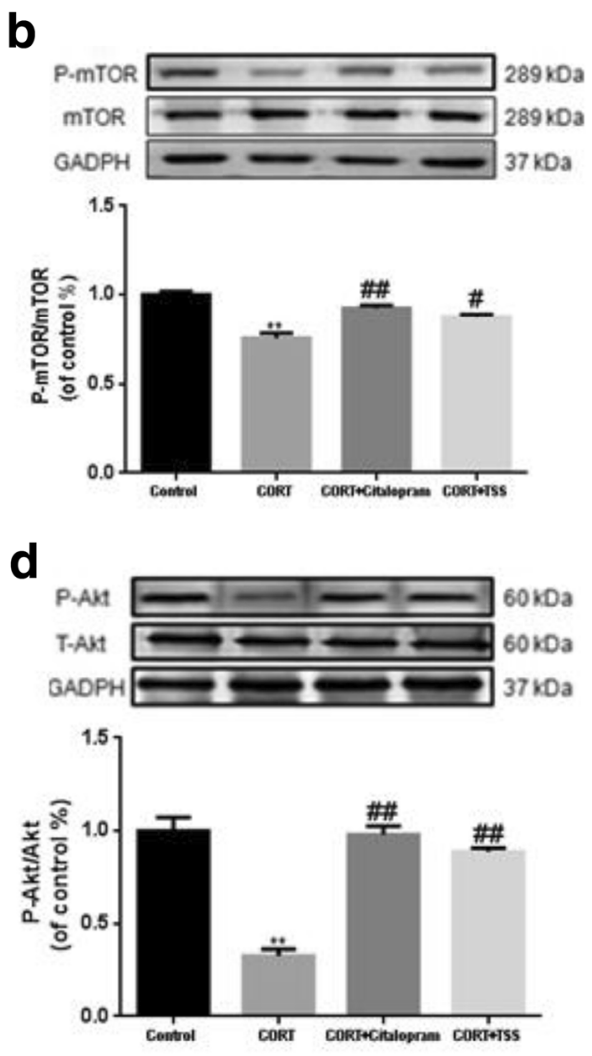

f
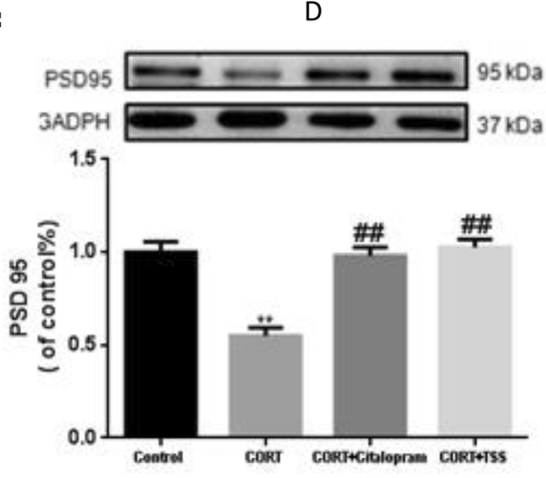

$\mathrm{F}$

Fig. 7 Representative western blot analysis of P-GluR1 (a), P-mTOR (b), P-ERK (c), P-Akt (d), synapsin-1(e) and PSD-95 (f) in the hippocampus after TSS $(25 \mathrm{mg} / \mathrm{kg})$ and citalopram $(10 \mathrm{mg} / \mathrm{kg})$ treatment in chronic corticosterone-treated mice. Data were analyzed by one-way ANOVA followed by Student-Newman-Keuls test. Each bar represents the mean \pm SEM. ${ }^{* *} P<0.01$, as compared with the control group and ${ }^{\# \#} P<0.01$, ${ }^{\#} P<0.05$, as compared with the CORT group

such as stimulation of general motor. Whereas, the mice treated with citalopram increased the distance moved and velocity, which is in line with previous study [26]. Taken together, TSS and citalopram exerted anxiolytic and antidepressant-like effects in chronic CORT treatment model. Our preliminary experiment indicated treatment with TSS produced a significant increase of 5HT level in the brain as compared to chronic mild stress model in mice. Increasing the 5-HT level in the brain may be the common path of antidepressant-like effect of citalopram and TSS. Glucocorticoid receptor (GR) mediates the expression of the serotonin transporter (SERT) and increases the 5-HT level in the brain. We have previous shown that Saikosaponins $\mathrm{D}$, the main active ingredient of TSS, up-regulated the GR protein expression in corticosterone-induced PC12 cells. This result 


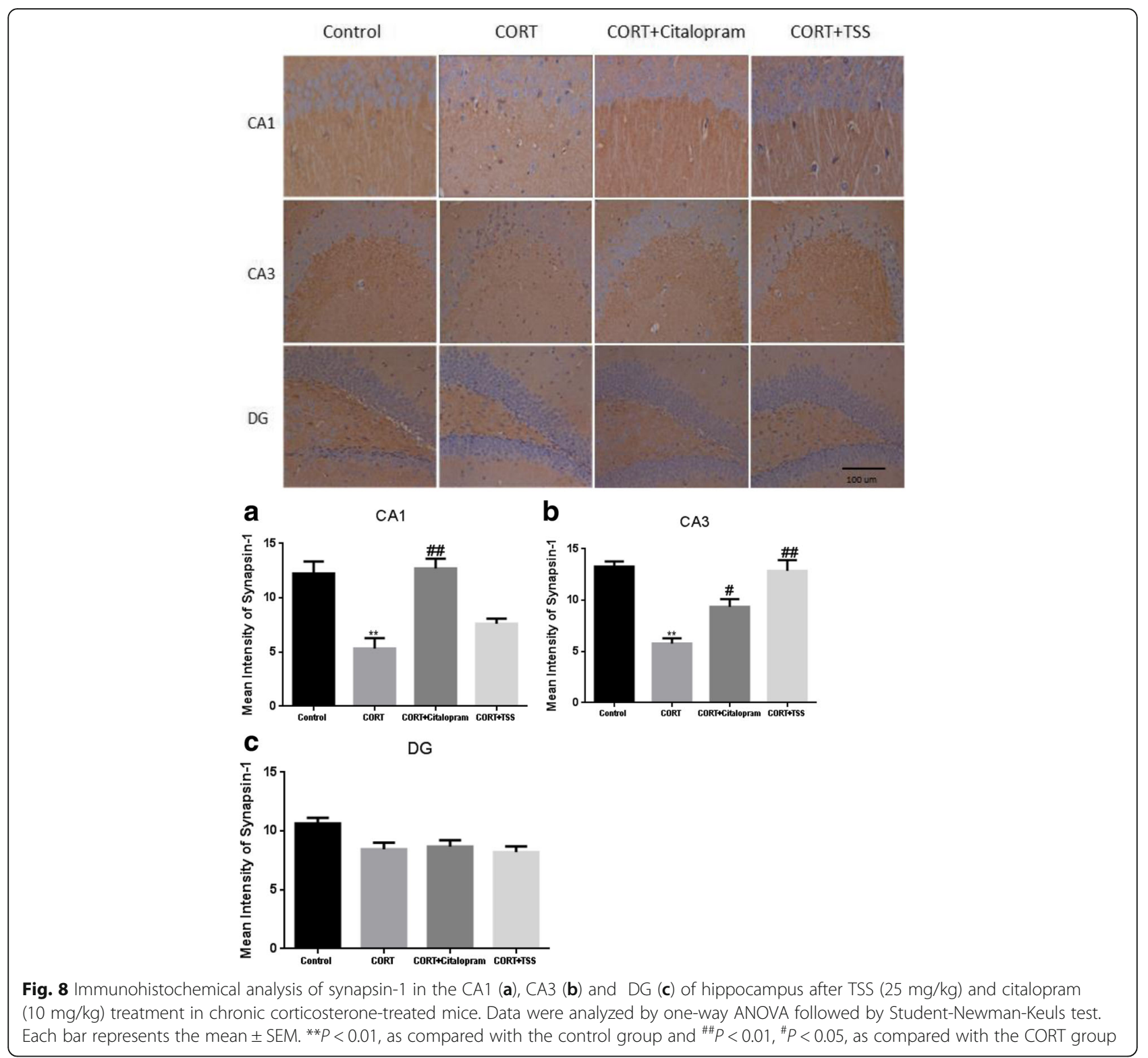

suggests that TSS induced increasing 5-HT level in the brain may be mediated by activating GR [14]. In the subsequent study, we should analyze the alteration of GR in the brain in the chronic corticosterone treatment model and investigate the effect of TSS on the GR expression.

It has been postulated that synaptic plasticity and synaptogenesis play an important role in the pathogenesis of depression and mechanisms of action of current antidepressant drugs [3]. Synapsin-1 is a presynaptic marker and is widely used for measurement of synaptic density [27]. PSD-95 is a post synaptic marker and is involved in glutamatergic synaptogenesis [28]. To detect whether costicosterone decreased the synaptic proteins level, synapsin-1 and PSD-95 levels were assessed in the hippocampus of chronic corticosterone-treated mice. Our present western blotting analysis shows that chronic treatment costicosterone decreases synapsin-1 and PSD-95 level in mice. TSS and citalopram treatment shows an increase in synapsin-1 and PSD-95 levels in the hippocampus of mice exposed to 5 week CORT treatment. To reveal localized changes of synaptic density in the hippocampus, immunohistochemical analysis was used to determine synapsin-1 expression in sub-regions of hippocampus in CORT-treated mice. The present study shows that 5 week CORT treatment induces reduction in synapsin-1level in the CA1 and CA3 of hippocampus. This finding supported previous work which exhibited prolonged corticosterone exposure decreased dendritic spine densities in the hippocampus CA1 
and CA3 [29]. In the previous studies, saikosaponin treatment induced molecular changes in the CA3 of hippocampus in the depression models [30,31]. Consistent with these findings, we found TSS induced a pronounced increase of synapsin-1 expression in the CA3 of hippocampus. Citalopram increased synapsin- 1 level both in the CA1 and CA3 region are in agreement with previous study showing that citalopram reversed the decreased synaptic plasticity in the hippocampus in the social isolation model [32]. These results suggest that TSS and citalopram might increase synaptic proteins expression and promote synaptogenesis in the hippcampus. Based on the above mentioned data, the mechanisms by which TSS and citalopram exert their antidepressant-like effects maybe involve both monoamine neurotransmission and synaptogenesis. Further studies should be performed to confirm this hypothesis.

Accumulating evidence shows that AMPA glutamatergic activation triggers the synaptogenic process in important regions such as the prefrontal cortex and hippocammpus [7]. In addition, several research results have shown that phosphorylation of mTOR and kinases ERK and Akt, is important mechanism underlying the activation of AMPA receptors in synaptogenesis [33-35]. Therefore, AMPA receptor-mTOR signaling pathway may be involved in the synaptic alteration underlying depression. To determine whether the antidepressant and anxiolytic like effects of TSS was accompanied by the activation of AMPA receptor-mTOR signaling pathway, the phosphorylation of GluR1 Ser845 (AMPA receptor subunit), mTOR and mTOR upstream regulator proteins (ERK and Akt) were assessed in chronic corticosterone-treated mice. Our research showed that TSS reversed the decreased level of P- GluR1 (ser845) induced by 5 week CORT administration. Simultaneously, 5 week CORT administration decreased the expression level of p-mTOR, p-ERK and p-Akt in the hippocampus, which was reversed by TSS and citalopram treatment. This finding of citalopram is in agreement with previous study showing that activation of the mTOR signaling was implicated in the antidepressant-like effect of subchronic citalopram treatment in the chronic mild stress model [36]. Our another study showed that pretreatment with 2,3-dioxo-6-nitro-1,2,3,4-tetrahydrobenzo (f) quinoxaline-7-sulfonamide (NBQX), an AMPA receptor antagonist, blocked the TSS induced antidepressant-like effects of decreased immobility time in the FST (data not shown). These results indicated that AMPA receptor-mTOR signaling pathway may be involved in the antidepressant-like effect of TSS. Further research is needed to examine whether pretreatment with NBQX would affect the activity of the mTOR signaling pathway ( $\mathrm{p}-\mathrm{mTOR}, \mathrm{p}-\mathrm{ERK}$ and p-Akt) after TSS treatment.

\section{Conclusion}

The present study indicates that repeated administration of TSS exerts antidepressant-like and anxiolytic effects in chronic CORT administration mice. Our data provide evidence that TSS improves the depressive and anxiety like behavior via induction of AMPA receptor and subsequent mTOR signaling pathway which are involved in synaptogenesis. Together with our previous research, TSS could be a potential efficacious candidate as a new therapy for depression.

\section{Abbreviations}

AMPA: A-amino-3-hydroxy-5-methyl-4-isoxazolepropionic acid; CORT: Corticosterone; EPM test: Elevated plus maze test; FST: Forced swim test; NSFT: Novelty suppressed feeding test; OFT: Open field test; TSS: Total saikosaponins

\section{Acknowledgements}

Not applicable.

\section{Funding}

This work was financially supported by Beijing Natural Science Foundation (grant no.7142105) and National Natural Science Foundation of China (grant no.31301890).

\section{Availability of data and materials}

The radices of Bupleurum yinchowense were purchased from Dingxi County, Gansu Province, China, June 2013, and authenticated by Prof. Bengang Zhang, the Institute of Medicinal Plant, Chinese Academy of Medical Sciences and Peking Union Medical College, Beijing, China, where a voucher specimen (No. 20130620) has been kept in Herbarium of the institute. The raw data and materials during the current study are available from the corresponding author on reasonable request.

Authors' contributions

XS designed the study, performed the experiment, did statistical analysis and wrote the manuscript. XL conducted the animal behavior tests. RP provided TSS. QW provided technical supports. YX conducted immunohistochemistry experiment. MS supervised the study. All authors read and approved the final manuscript.

Ethics approval and consent to participate

The experiments were approved by the ethical committee for the use of experimental animals of the Institute of Laboratory Animal Sciences (No: ILAS-PG-2015-011).

Consent for publication

Not applicable.

Competing interests

The authors declare that they have no competing interests.

\section{Publisher's Note}

Springer Nature remains neutral with regard to jurisdictional claims in published maps and institutional affiliations.

\footnotetext{
Author details

${ }^{1}$ Comparative Medical Center, Beijing Key Laboratory for Animal Models of Emerging and Reemerging Infectious Diseases, Peking Union Medical College (PUMC) \& Institute of Laboratory Animal Science, Chinese Academy of Medical Science(CAMS), PanjiayuanNanli No. 5, Beijing 100021, China. ${ }^{2}$ Institute of Medicinal Plant Development, Chinese Academy of Medical Science, Peking Union Medical College, No 151, North Road Malianwa, Haidian District, Beijing 100193, China. ${ }^{3}$ Prelinical Medicine Research Center/ School of Pharmacy, Southwest Medical University, Zhongshan Road, 3-319, Jiangyang district, Luzhou 646000, Sichuan Provience, China.
} 
Received: 25 May 2017 Accepted: 22 March 2018

\section{Published online: 02 April 2018}

\section{References}

1. Yan $\mathrm{H}$, Min H. Study of prescriptions containing Bupleurum as monarch for treatment of depression. Chin J Exp Tradit Med Formulae. 2010;17:247-9.

2. Sun X, SZ, Li T, Pan R, Liu X, Bu L, Kong L. Antidepressant-like effects of total saikosaponins of Bupleurum yinchowense in mice. J Med Plants Res. 2012; 6(26):4308-16.

3. Duman RS, Aghajanian GK. Synaptic dysfunction in depression: potential therapeutic targets. Science. 2012;338(6103):68-72.

4. Zhang H, Etherington LA, Hafner AS, Belelli D, Coussen F, Delagrange P, Chaouloff F, Spedding M, Lambert JJ, Choquet D, et al. Regulation of AMPA receptor surface trafficking and synaptic plasticity by a cognitive enhancer and antidepressant molecule. Mol Psychiatry. 2013;18(4):471-84.

5. Walker AK, Budac DP, Bisulco S, Lee AW, Smith RA, Beenders B, Kelley KW, Dantzer R. NMDA receptor blockade by ketamine abrogates lipopolysaccharide-induced depressive-like behavior in C57BL/6J mice. Neuropsychopharmacology. 2013;38(9):1609-16.

6. Akinfiresoye L, Tizabi Y. Antidepressant effects of AMPA and ketamine combination: role of hippocampal BDNF, synapsin, and mTOR. Psychopharmacology. 2013;230(2):291-8.

7. Ignacio ZM, Reus GZ, Arent CO, Abelaira HM, Pitcher MR, Quevedo J. New perspectives on the involvement of mTOR in depression as well as in the action of antidepressant drugs. Br J Clin Pharmacol. 2015;82(5):1280-90.

8. Zhou W, Wang N, Yang C, Li XM, Zhou ZQ, Yang JJ. Ketamine-induced antidepressant effects are associated with AMPA receptors-mediated upregulation of mTOR and BDNF in rat hippocampus and prefrontal cortex. Eur Psychiatry. 2014;29(7):419-23.

9. Chen KT, Tsai MH, Wu CH, Jou MJ, Wei IH, Huang CC. AMPA receptor-mTOR activation is required for the antidepressant-like effects of sarcosine during the forced swim test in rats: insertion of AMPA receptor may play a role. Front Behav Neurosci. 2015;9:162.

10. Swaab DF, Bao AM, Lucassen PJ. The stress system in the human brain in depression and neurodegeneration. Ageing Res Rev. 2005;4(2):141-94.

11. Le Dantec Y, Hache G, Guilloux JP, Guiard BP, David DJ, Adrien J, Escourrou P. NREM sleep hypersomnia and reduced sleep/wake continuity in a neuroendocrine mouse model of anxiety/depression based on chronic corticosterone administration. Neuroscience. 2014;274:357-68.

12. Crupi R, Mazzon E, Marino A, La Spada G, Bramanti P, Cuzzocrea S, Spina E. Melatonin treatment mimics the antidepressant action in chronic corticosterone-treated mice. J Pineal Res. 2010;49:123-9.

13. Tse YC, Bagot RC, Wong TP. Dynamic regulation of NMDAR function in the adult brain by the stress hormone corticosterone. Front Cell Neurosci. 2012;6:9.

14. Li Z-Y, Guo Z, Liu Y-M, Liu X-M, Chang Q, Liao Y-h, Pan R-L. Neuroprotective effects of Total Saikosaponins of Bupleurum yinchowense on corticosterone-induced apoptosis in PC12 cells. J Ethnopharmacol. 2013; 148(3):794-803.

15. Porsolt R, Bertin A, Jalfre M. Behavioral despair in mice: a primary screening test for antidepressants. Arch Int Pharmacodyn Ther. 1977;229(2):327-36.

16. Prut $L$, Belzung $C$. The open field as a paradigm to measure the effects of drugs on anxiety-like behaviors: a review. Eur J Pharmacol. 2003;463(1-3):3-33.

17. Calabrese EJ. An assessment of anxiolytic drug screening tests: hormetic dose responses predominate. Crit Rev Toxicol. 2008;38(6):489-542.

18. Stedenfeld KA, Clinton SM, Kerman IA, Akil H, Watson SJ, Sved AF. Noveltyseeking behavior predicts vulnerability in a rodent model of depression. Physiol Behav. 2011;103(2):210-6.

19. Yang J-S, Zhang N, Song M-J, Sun X-P. Antidepressant-like effect and learning and memory improvement of Bupleurum yinchowense total saikosaponins and its possible mechanism in mice. Chin J Exp Tradit Med Formulae. 2016;24(22):134-9.

20. Cai Z-Z, Xu G-Y, Dong H-Y. Protective effect of Saikosaponin on the hippocampal neuron of depression model rats. Med Innov China. 2016; 13(03):28-30.

21. Darcet F, Mendez-David I, Tritschler L, Gardier AM, Guilloux JP, David DJ. Learning and memory impairments in a neuroendocrine mouse model of anxiety/depression. Front Behav Neurosci. 2014;8:136

22. Quesseveur G, Portal B, Basile JA, Ezan P, Mathou A, Halley H, Leloup C, Fioramonti X, Deglon N, Giaume C, et al. Attenuated levels of hippocampal Connexin 43 and its phosphorylation correlate with antidepressant- and anxiolytic-like activities in mice. Front Cell Neurosci. 2015;9:490.
23. Hirschfeld RMA. The comorbidity of major depression and anxiety disorders: recognition and Management in Primary Care. Prim Care Companion J Clin Psychiatry. 2001;3(6):244-54.

24. Wu Z, Fang Y. Comorbidity of depressive and anxiety disorders: challenges in diagnosis and assessment. Shanghai Arch Psychiatry. 2014;26(4):227-31.

25. Coplan JD, Aaronson CJ, Panthangi V, Kim Y. Treating comorbid anxiety and depression: psychosocial and pharmacological approaches. World J Psychiatry. 2015:5(4):366-78.

26. Kellner M, Porseryd T, Hallgren S, Porsch-Hallstrom I, Hansen SH, Olsen KH. Waterborne citalopram has anxiolytic effects and increases locomotor activity in the three-spine stickleback (Gasterosteus aculeatus). Aquat Toxicol. 2016;173:19-28.

27. Grizzell JA, larkov A, Holmes R, Mori T, Echeverria V. Cotinine reduces depressive-like behavior, working memory deficits, and synaptic loss associated with chronic stress in mice. Behav Brain Res. 2014:268(0):55-65.

28. Renard J, Vitalis T, Rame M, Krebs M-O, Lenkei Z, Le Pen G, Jay TM. Chronic cannabinoid exposure during adolescence leads to long-term structural and functional changes in the prefrontal cortex. Eur Neuropsychopharmacol. 2016;26(1):55-64.

29. Orlowski D, Elfving B, Muller HK, Wegener G, Bjarkam CR. Wistar rats subjected to chronic restraint stress display increased hippocampal spine density paralleled by increased expression levels of synaptic scaffolding proteins. Stress. 2012;15(5):514-23.

30. Du Y-W, Wang Y-L, Yin L, Zhao X-M, Qin S-L, Zhao Y-L. Effects of CHSG powder on ERK and $P$ - ERK in Hippocampus of rats with liver - qi stagnation. Chin Arch Tradit Chin Med. 2012:30(06):1367-9.

31. Zhang X-J, Dong H-Y, Sun Y-R, Lin C-R, Li X-Y. Effect of ChaihuShugan powder on apoptosis in hippocampal CA3 area of Depressive rats. Lishizhen Med Materia Medica Res. 2011;22(01):43-5.

32. Gong WG, Wang YJ, Zhou H, Li XL, Bai F, Ren QG, Zhang ZJ. Citalopram ameliorates synaptic plasticity deficits in different cognition-associated brain regions induced by social isolation in middle-aged rats. Mol Neurobiol. 2017;54(3):1927-38

33. Voleti B, Navarria A, Liu RJ, Banasr M, Li N, Terwilliger R, Sanacora G, Eid T, Aghajanian G, Duman RS. Scopolamine rapidly increases mammalian target of rapamycin complex 1 signaling, synaptogenesis, and antidepressant behavioral responses. Biol Psychiatry. 2013;74(10):742-9.

34. Li N, Lee B, Liu RJ, Banasr M, Dwyer JM, Iwata M, Li XY, Aghajanian G, Duman RS. mTOR-dependent synapse formation underlies the rapid antidepressant effects of NMDA antagonists. Science. 2010;329(5994):959-64.

35. Dwyer JM, Duman RS. Activation of mammalian target of rapamycin and synaptogenesis: role in the actions of rapid-acting antidepressants. Biol Psychiatry. 2013;73(12):1189-98.

36. Opal MD, Klenotich SC, Morais M, Bessa J, Winkle J, Doukas D, Kay LJ, Sousa N, Dulawa SM. Serotonin 2 receptor antagonists induce fast-onset antidepressant effects. Mol Psychiatry. 2014;19(10):1106-14.

\section{Submit your next manuscript to BioMed Central and we will help you at every step:}

- We accept pre-submission inquiries

- Our selector tool helps you to find the most relevant journal

- We provide round the clock customer support

- Convenient online submission

- Thorough peer review

- Inclusion in PubMed and all major indexing services

- Maximum visibility for your research

Submit your manuscript at www.biomedcentral.com/submit
Biomed Central 